\title{
GROUND FILTERING LiDAR DATA BASED ON MULTI-SCALE ANALYSIS OF HEIGHT DIFFERENCE THRESHOLD
}

\author{
Pejman Rashidi a , Heidar Rastiveis a, *

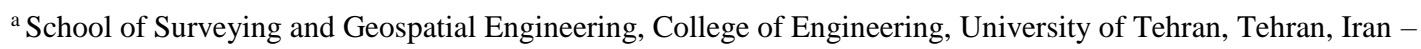 \\ (pejman.rashidi,hrasti)@ut.ac.ir
}

KEY WORDS: DTM, LiDAR Point Clouds, Filtering

\begin{abstract}
:
Separating point clouds into ground and non-ground points is a necessary step to generate digital terrain model (DTM) from LiDAR dataset. In this research, a new method based on multi-scale analysis of height difference threshold is proposed for ground filtering of LiDAR data. The proposed method utilizes three windows with different sizes in small, average and large to cover the entire LiDAR point clouds, then with a height difference threshold, point clouds can be separated to ground and non-ground in each local window. Meanwhile, the best threshold values for size of windows are considered based on physical characteristics of the ground surface and size of objects. Also, the minimum of height of object in each window selected as height difference threshold. In order to evaluate the performance of the proposed algorithm, two datasets in rural and urban area were applied. The overall accuracy in rural and urban area was $96.06 \%$ and $94.88 \%$ respectively. These results of the filtering showed that the proposed method can successfully filters non-ground points from LiDAR point clouds despite of the data area.
\end{abstract}

\section{INTRODUCTION}

In recent decades, airborne light detection and ranging (LiDAR) has been demonstrated to be useful for rapidly gathering three-dimensional coordinates of ground and nonground objects (Rabbani and Van Den Heuvel 2005; Rabbani et al. 2007; Filin and Pfeifer 2006). Meanwhile, LiDAR has several advantages in comparison with traditional field surveying and photogrammetric mapping, e.g., cost-effective over a large area for acquisition of vertical information, higher accuracy and gathering information in any kinds of weather (Meng et al. 2009; Li et al. 2014).

One of the important products of LiDAR is digital terrain model (DTM) of the bare earth (Rottensteiner et al. 2002), which is the three-dimensional model indicating the spatial distribution of the earth's surface (Quan et al. 2016). In DTM generation from LiDAR data, the first step is separating ground and non-ground points, a process referred to as filtering (Li 2013).

In the past two decades, many DTM generation methods have been developed, but there are a lot of challenges for filtering LiDAR data yet. The most important features that can be confused some ground filtering algorithms involve shrubs, bridges and complex mixed covering (Meng, Currit, and Zhao 2010).

In order to identify ground points, there are some of approaches that work with the either raw LiDAR point clouds or gridded elevation values (Sithole and Vosselman 2004). Work with the either raw LiDAR point clouds have certain advantages such as require less preprocessing, and avoid errors introduced by interpolation (Meng et al. 2009).

Divers of popular filtering LiDAR data methods have been proposed at present. The number of filtering algorithms are based on the slope of a line segment between neighboring points (Wang and Tseng 2010). Feng et al. (2009) proposed a filtering algorithm that combines slope-based and region- growing methods for filtering LiDAR point clouds in urban area. However, their results for this combined approach is not guaranteed to function well in dense urban areas.

Some of filtering algorithms, search for neighboring points by creating a Triangulated Irregular Network (TIN) with certain constraints of angle and distance (Uysal and Polat 2014). This algorithm assumes that bare ground areas are usually smooth surfaces without sharp corners in terrain surfaces (Meng, Currit, and Zhao 2010). Axelsson (2000) developed an active-TIN based model and utilizes a TIN surface to iteratively estimate ground surface. The algorithm steadily removes non-ground points based on the elevation difference and angle to the closest triangle.

There are a number of algorithms based on morphological approaches for filtering LiDAR point clouds. In this algorithm, typical processes include opening, closing, dilatation and erosion based on kernel operators (Liu 2008). Arefi and Hahn (2005) developed a morphological method based on geodesic dilation and changing window sizes to gradually remove non-ground points. Their results show that the selection of window sizes is critical to removing objects with different sizes.

From the aforementioned studies, it can be concluded that although several methods have been proposed for filtering of LiDAR points cloud, a powerful method that can completely eliminate all objects from LiDAR data has not yet been developed. Therefore, LiDAR data filtering can be known as an open problem in Photogrammetry and Remote Sensing. In this study, we propose a new method based on multi-scale analysis of height difference threshold on raw LiDAR point clouds for filtering of LiDAR data. Different size of window includes three windows, the first one could remove small non-ground objects such as shrubs, but the second window that is average of small and large window, eliminates medium size objects, such as vehicles. Also, the large window can remove large non-ground objects, such as buildings. According to the physical characteristics of the 
ground surface and size of objects, the best threshold value is considered. In the following, the paper explains the basic procedure of this algorithm and presents results and analyses obtained from its implementation.

\section{PROPOSED METHOD}

In this research, a new method for filtering of LiDAR point clouds based on multi-scale analysis on raw LiDAR data is proposed. The flowchart of this method is shown in Figure. 1. As can be seen from this figure, after removing outliers in pre-processing, some parameters are calculated. These parameters include size of windows and height difference threshold. After that, filtering is completed through three main steps: small, average and large window size. The main steps of the proposed method are described in the following sections.

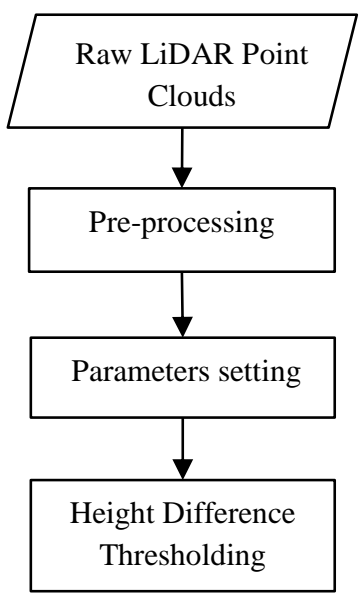

Figure. 1. Flowchart of the proposed method for filtering of LiDAR point clouds.

\subsection{Pre-processing}

In this study, in the pre-processing step, outliers should be removed from the data. In LiDAR data, the outliers are the points with abnormal elevation values, either higher or lower than the surrounded points. High outliers, which usually include random errors and can be resulted by birds or airplanes, usually can be eliminated during the filtering process, because they can be assumed as non-ground objects (Meng et al. 2009). While, low outliers are below the earth surface and may be resulted from several times reflecting of laser returns. These outliers may seriously affect the filtering results and, therefore, they should be removed from the data in the pre-processing step (Li 2013).

In this proposed method, a local window considered and as can be seen in Equation (1) the gray values $G_{i}^{p} \in G^{p} . i \in$ $1 \ldots n$ are sorted by a function $s$.

$$
G_{s(1)}^{p} \leq \cdots \leq G_{s(n)}^{p}
$$

After sorting the gray values, all of points compare with average of point gray values. Then, the points that are lower than average with regards to a predefined threshold, could potentially be outliers and would be removed from data.

\subsection{Parameters Setting}

After pre-processing step, some parameters are calculated for identify diverse of objects. As is clear, in LiDAR data there are lots of objects with different sizes and heights. Therefore, we should have enough information from objects for filtering LiDAR data.

In order to find types of objects, three windows with different sizes and height thresholds are considered. The first window is small window and is appropriate for removing small objects like trees and shrubs, so the size of small window is usually the same for all of datasets. The last window is large window and is used for removing large objects such as, buildings and bridges. The size of large window is based on the largest object in LiDAR data. The second window is average window and is appropriate for identify objects between small and large sizes, such as vehicles. The size of average window is based on average between large and small window sizes.

In proposed method, for filtering LiDAR point clouds in urban and rural area, there is one height threshold for each size of window. In this study, the minimum of height of object in each window selected as height difference threshold. Therefore, in each window, size of window and height difference threshold should be calculated.

\subsection{Height Difference Thresholding}

In this study, for filtering LiDAR point clouds, the elevation difference between all points in local window and the minimum elevation point is calculated, so further height compared to their neighbors range, are removed in the window thresholding step. The main steps of the filtering are described in more details in Figure. 2.

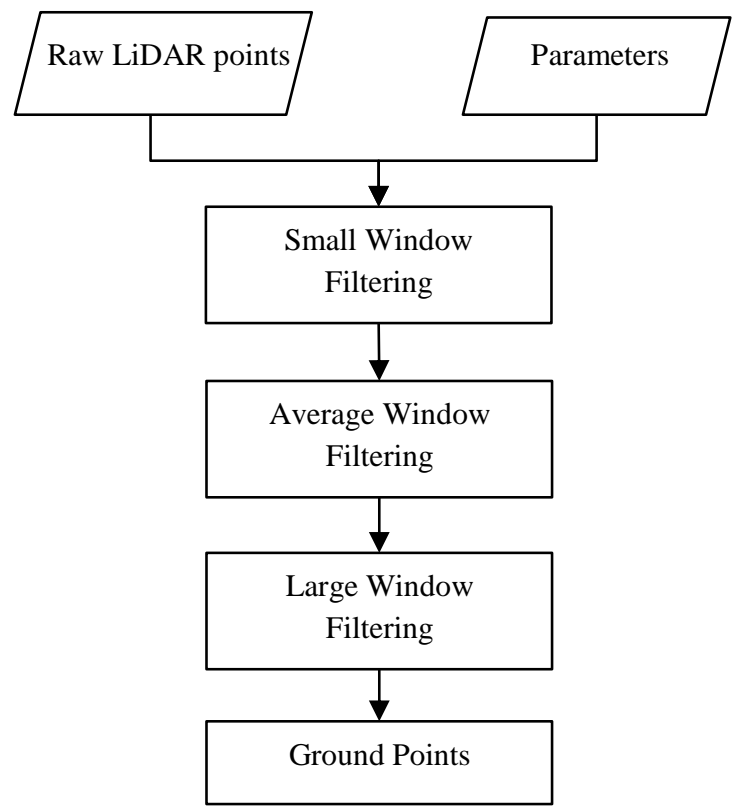

Figure. 2. Multi-Scale Analysis of height difference theresholding.

\section{Data}

In this study, to evaluate the performance of the proposed method, two datasets with different spatial resolution in rural 
and urban area have been tested. The details of these datasets are described in the following sections.

\subsection{Rural Dataset}

The first dataset is the benchmark dataset provided by the International Society for Photogrammetry and Remote Sensing (ISPRS) Commission III/WG3 (http://www.itc.nl/isprswgIII-3/filtertest/). This paper chooses the sample_61 for testing the algorithm on the rural area. In addition, the reference dataset had been provided by ISPRS using semi-automatic and manually filtering with recognition landscape and aerial images (Chen et al. 2013) This sample includes 35060 points with vegetation and a large gap in data. Meanwhile, this sample is selected as low resolution data with small vegetation. Also, there are ground surface changes in this area. LiDAR point clouds of this sample is shown in Figure. 3.

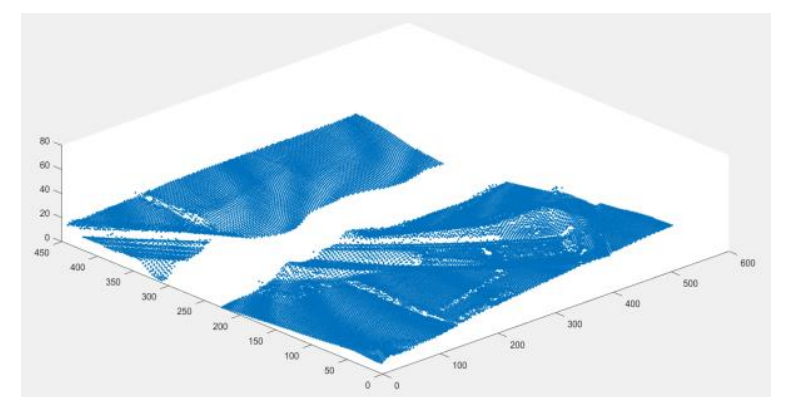

Figure. 3. LiDAR points cloud from ISPRS with low resolution

\subsection{Urban Dataset}

In this study, to evaluate better of algorithm, a new dataset with high resolution in urban area is selected. This dataset was provided by (IEEE 2015), and is cropped a part of urban area in Zeebruges, Belgium with an average point density of 65 points $/ \mathrm{m}^{2}$, which is related to point spacing of approximately $10 \mathrm{~cm}$. Also, the ground truth for this sample was manually generated by an expert. LiDAR points cloud of this sample is shown in Figure. 4. As one can observe from this figure, this dataset covers various terrain types including irregularly shaped buildings with eccentric roofs, roads, vehicles and vegetation.

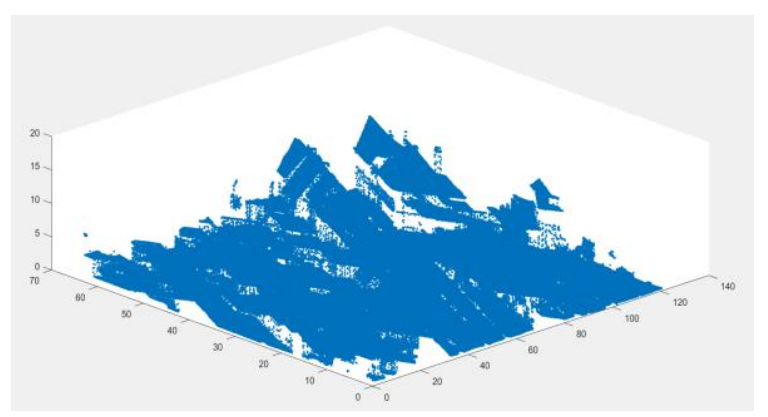

Figure. 4. LiDAR points cloud from IEEE with high resolution

\section{EXPERIMENTS AND PERFORMANCE EVALUATION}

In this study, the proposed method implemented using MATLAB R2015b. In the following, the results have been discussed and evaluated.

\subsection{Validation of the filtering}

The proposed algorithm was tested by ISPRS and IEEE datasets to evaluate the performance of the method in rural and urban areas. First of all, in pre-processing step, low outliers removed from LiDAR point clouds. For this purpose, a window based on roving on all points considered on raw LiDAR data with size of 10 meter. Then, in each local window, after sorting the gray values, the points that are lower than average with regards to a predefined threshold that is 5 meter in rural area and 10 meter in urban area, could potentially be outliers and would be removed from data.

For next steps, two parameters were used for testing the algorithm including the window size and the height difference threshold values for each of small, average and large windows. Table 1 summarizes the applied parameters in testing the algorithm using both datasets.

\begin{tabular}{ccc}
\hline \hline Parameters & $\begin{array}{c}\text { ISPRS } \\
\text { Data }\end{array}$ & $\begin{array}{c}\text { IEEE } \\
\text { Data }\end{array}$ \\
\hline $\begin{array}{c}\text { Size of small } \\
\text { window }\end{array}$ & $3.6 \mathrm{~m}$ & $7 \mathrm{~m}$ \\
Height threshold in small \\
window \\
Size of average \\
$\quad$ window \\
$\begin{array}{c}\text { Height threshold in average } \\
\text { window } \\
\text { Size of } \\
\text { large window }\end{array}$ & $2 \mathrm{~m}$ & $0.5 \mathrm{~m}$ \\
Height threshold in large \\
window
\end{tabular}

Table1. Parameter values for filtering raw LiDAR data.

In this research, to evaluate the efficiency of the proposed method, three indexes of error type I, error type II and accuracy based on a simple confusion matrix were used. These equations can be observed in Equations (2), (3) and (4). The structure of confusion matrix is showed in Table 2.

\begin{tabular}{|c|c|c|c|}
\hline \multirow{5}{*}{ 芯 } & & \multicolumn{2}{|c|}{ Classified Points } \\
\hline & & $\begin{array}{l}\text { Ground Points } \\
\text { Points }\end{array}$ & Non-ground \\
\hline & Ground Points & & \\
\hline & & $\mathrm{a}$ & b \\
\hline & Points & $\mathrm{c}$ & d \\
\hline
\end{tabular}

Table2. Structure of Confusion Matrix 
error type $\mathrm{I}=b /(a+b)$

error type $\mathrm{II}=c /(c+d)$

accuracy $=(a+d) /(a+b+c+d)$

The results of the proposed filtering method are shown in Figure. 5. As can be seen in Figure. 5(a), the proposed method showed high performance in filtering LiDAR data in rural area, however, there are more dramatic ground surface changes in this area. Meanwhile, as can be observed in Figure. 5(b), the special objects such as, irregularly shaped buildings with eccentric roofs, vehicles and vegetation can be well filtered in urban area.

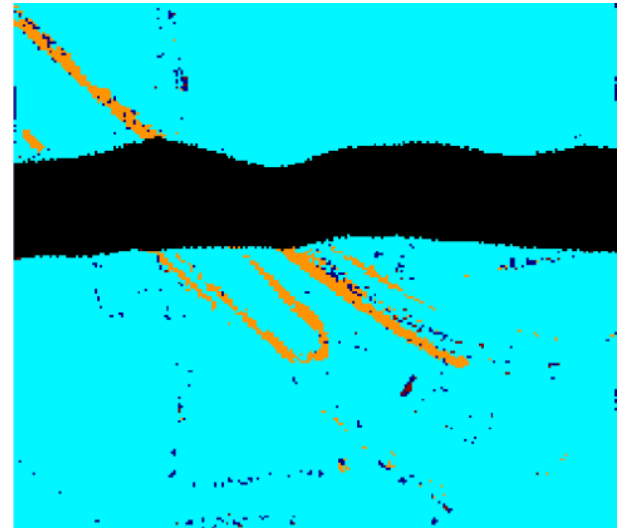

(a)

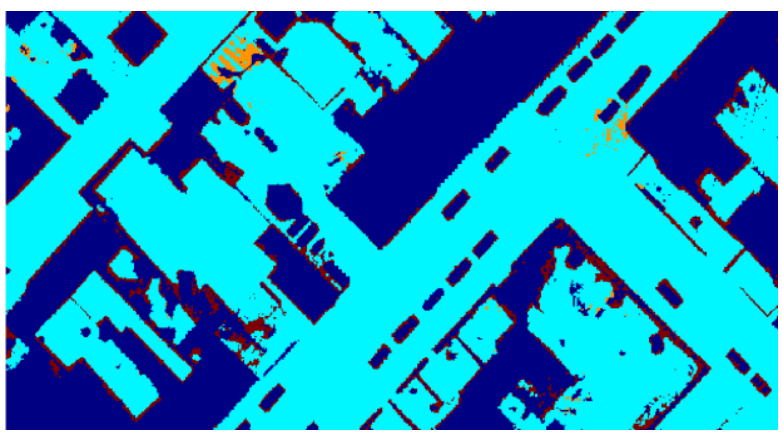

(b)

Ground

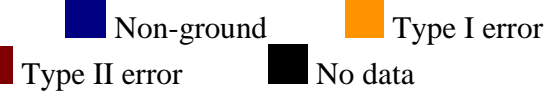

Figure. 5. Error distribution in filtering of the LiDAR datasets. (a) ISPRS, (b) IEEE

\subsection{Discuss and evaluate the results}

Table 3 shows the calculated type I, type II and accuracy for the test samples. This table vividly illustrates the proposed algorithm was successfully filtered these LiDAR datasets with low level resulted type I and type II error rates. However, type II error in ISPRS dataset was rarely high, because of there are more dramatic ground surface changes and some of points labeled incorrectly as ground points. Also, accuracy for both datasets are well, and it means proposed method has well effect on filtering LiDAR data, and it is robust to difference issues such as resolution of LiDAR data, different slopes and complexity of the objects

\begin{tabular}{cccc}
\hline \hline $\begin{array}{c}\text { Sample } \\
\text { Dataset }\end{array}$ & $\begin{array}{c}\text { Type I } \\
\text { Error(\%) }\end{array}$ & $\begin{array}{c}\text { Type II } \\
\text { Error(\%) }\end{array}$ & Accuracy \\
\hline ISPRS & 3.8 & 16.87 & 96.06 \\
IEEE & 6.52 & 4.43 & 94.88 \\
\hline \hline
\end{tabular}

Table3. Accuracy indexes for ISPRS dataset in the SPWT algorithm.

A lot of researchers have used the ISPRS dataset to evaluate their filtering algorithms. In this study, to quantitatively analyze the accuracy of the proposed algorithm the resulted accuracy of sample_61 in ISPRS dataset from the proposed method is compared to another filtering and are shown in Table 4.

\begin{tabular}{cc}
\hline \hline Author & Accuracy \\
\hline (Mongus and Žalik 2012) & 95.15 \\
(Li 2013) & 94.19 \\
(Zhang and Lin 2013) & 83.87 \\
(Hui et al. 2016) & 97.67 \\
Proposed Method & $\mathbf{9 6 . 0 6}$ \\
\hline \hline
\end{tabular}

Table4. Accuracy of sample_61 in ISPRS dataset reported by novel algorithms.

As can be seen from Table 4, the proposed method has good performance in LiDAR points cloud filtering in comparison with another novel method.

\section{CONCLUSION}

In this research, a new LiDAR point clouds filtering based on multi-scale analysis on raw LiDAR data was proposed. The proposed method, remove low outliers in pre-processing step and after calculate parameters for filtering, ground points can be detected by different size of windows and height difference threshold for each window. The proposed algorithm was tested by two different datasets in rural and urban area. In filtering of raw LiDAR data, the proposed method showed high performance, so that overall accuracy for rural area was $96.06 \%$ and $94.88 \%$ for urban area. The results of the filtering showed that the proposed method can successfully filters non-ground points from LiDAR point clouds despite of the data area.

The future research work is to try controlling the increment of the type II error due to it was a little big in rural area and find automatically the parameters of the proposed method to reduce the role of operator.

\section{REFERENCES}

Arefi, H. and M. Hahn (2005). "A morphological reconstruction algorithm for separating off-terrain points from terrain points in laser scanning data." International Archives of Photogrammetry, Remote Sensing and Spatial Information Sciences 36(3/W19): 120-125. 
Axelsson, P. (2000). "DEM generation from laser scanner data using adaptive TIN models." International Archives of Photogrammetry and Remote Sensing 33(B4/1; PART 4): 111-118.

Chen, D., et al. (2013). "A mathematical morphology-based multi-level filter of LiDAR data for generating DTMs." Science China Information Sciences: 1-14.

Feng, Y., et al. (2009). Urban DEM generation from airborne LiDAR data. Urban Remote Sensing Event, 2009 Joint, IEEE.

Filin, S. and N. Pfeifer (2006). "Segmentation of airborne laser scanning data using a slope adaptive neighborhood." ISPRS journal of Photogrammetry and Remote Sensing 60(2): 71-80

Hui, Z., et al. (2016). "An improved morphological algorithm for filtering airborne LIDAR point cloud based on multi-level kriging interpolation." Remote Sensing 8(1): 35.

IEEE (2015). "Image analysis and data fusion - GRSS | IEEE | Geoscience \& remote sensing society ".

Li, Y. (2013). "Filtering airborne LiDAR data by an improved morphological method based on multi-gradient analysis." Int. Arch. Photogr., Remote Sens. Spatial Inf. Sci 40: 191-194.

Li, Y., et al. (2014). "An improved top-hat filter with sloped brim for extracting ground points from airborne lidar point clouds." Remote Sensing 6(12): 12885-12908.

Liu, X. (2008). "Airborne LiDAR for DEM generation: some critical issues." Progress in Physical Geography 32(1): 31-49.

Meng, X., et al. (2010). "Ground filtering algorithms for airborne LiDAR data: A review of critical issues." Remote Sensing 2(3): 833-860.

Meng, X., et al. (2009). "A multi-directional ground filtering algorithm for airborne LIDAR." ISPRS journal of Photogrammetry and Remote Sensing 64(1): 117-124.

Mongus, D. and B. Žalik (2012). "Parameter-free ground filtering of LiDAR data for automatic DTM generation." ISPRS journal of Photogrammetry and Remote Sensing 67: $1-12$.

Quan, Y., et al. (2016). "Filtering LiDAR data based on adjacent triangle of triangulated irregular network." Multimedia Tools and Applications: 1-13.

Rabbani, T., et al. (2007). "An integrated approach for modelling and global registration of point clouds." ISPRS journal of Photogrammetry and Remote Sensing 61(6): 355370.

Rabbani, T. and F. Van Den Heuvel (2005). "Efficient hough transform for automatic detection of cylinders in point clouds." ISPRS WG III/3, III/4 3: 60-65.

Rottensteiner, F., et al. (2002). LIDAR activities at the Viennese institute of photogrammetry and remote sensing. Proceedings of the 3rd Int. LIDAR Workshop, Columbus.
Sithole, G. and G. Vosselman (2004). "Experimental comparison of filter algorithms for bare-Earth extraction from airborne laser scanning point clouds." ISPRS journal of Photogrammetry and Remote Sensing 59(1): 85-101.

Uysal, M. and N. Polat (2014). "Investigating Performance Of Airborne Lidar Data Filtering With Triangular Irregular Network (TIN) Algorithm." The International Archives of Photogrammetry, Remote Sensing and Spatial Information Sciences 40(7): 199.

Wang, C.-K. and Y.-H. Tseng (2010). DEM generation from airborne LiDAR data by an adaptive dual-directional slope filter, na.

Zhang, J. and X. Lin (2013). "Filtering airborne LiDAR data by embedding smoothness-constrained segmentation in progressive TIN densification." ISPRS journal of Photogrammetry and Remote Sensing 81: 44-59. 